\section{Dr. Favalli, et al reply}

\section{To the Editor:}

We thank Dr. So, et al $l^{1}$ for the interest in our letter ${ }^{2}$ and for sharing the results about the coronavirus disease 2019 (COVID-19) in patients with systemic lupus erythematosus (SLE) in Hong Kong ${ }^{1}$.

We agree that the quantification of the risk of infection with severe acute respiratory coronaviruses-2 (SARS-CoV-2) in patients with SLE is a major concern. This is even more true in light of the recently published data from Mathian and colleagues ${ }^{3}$, who analyzed the course of COVID-19 in a case series of 17 patients with SLE. Of these, 13 (76\%) developed interstitial pneumonia, complicated by respiratory failure in 11 (65\%) and acute respiratory distress syndrome in 5 (29\%). Moreover, 3 patients had acute renal failure, with 2 patients requiring hemodialysis. The results of this analysis first demonstrated that the clinical course of COVID-19 in patients with SLE seems to be particularly unfavorable and facilitated by multiorgan involvement and the several comorbidities often complicating this complex autoimmune disease. Because of this evidence, it becomes even more crucial to define whether patients with SLE are burdened with an increased risk of contracting COVID-19 to efficiently establish a prophylaxis program. Another still critical point is the definition of the role of chloroquine and hydroxychloroquine in the prevention and treatment of SARS-CoV-2 infection. In fact, after the in vitro demonstration of antimalarials' ability to interfere with the endocytosis of the virus within the target cells and the first favorable in vivo results that led to their inclusion in COVID-19 management protocols worldwide ${ }^{4}$, the most recent clinical studies have provided controversial results that leave many doubts about the real efficacy and safety profile of these drugs for this indication ${ }^{5}$. The data we recently published on the frequency of COVID-19 in connective tissue diseases ${ }^{2}$, investigated through an exploratory survey to evaluate the incidence and course of COVID-19, included a portion of patients with SLE, which has further increased over time as our data collection progressed. In the most recent update, the group under review included 65 patients with SLE, none of whom received a diagnosis of confirmed COVID- $19^{6}$. Conversely, in a cohort of 165 patients with SLE investigated by telemedicine in another rheumatology unit in Lombardy, 4 patients were diagnosed with COVID-19 confirmed by swab and 8 with highly suspicious COVID-19 (at least 3 out of 4 symptoms among fever, dyspnea, cough, and dysgeusia/anosmia plus established contact with a COVID-19 patient, no swab test performed) 7 . However, these study populations are still too limited in number to draw definitive conclusions, and larger cohort studies are certainly warranted to address the issue. With this in mind, the COVID-19 Global Rheumatology Alliance recently launched a worldwide register for patients with rheumatic diseases with COVID-19, which may decisively contribute with additional findings. Preliminary data recently published on 110 patients also included 19 SLE cases, confirming that the infection can certainly affect this type of patients as well'.

It is very likely that patients with SLE are exposed to at least the same risk as the general population of acquiring COVID-19; however, they generally have a more unfavorable clinical course.
Ennio Giulio Favalli ${ }^{1}$, MD

Elena Agape ${ }^{1}$, MD

Roberto Caporali ${ }^{1,2}, \mathrm{MD}$

${ }^{1}$ Division of Clinical Rheumatology, Azienda Socio

Sanitaria Territoriale (ASST) Gaetano Pini-CTO Institute;

${ }^{2}$ Department of Clinical Sciences and Community Health, Research Center for Adult and Pediatric Rheumatic Diseases, Università degli Studi di Milano, Milan, Italy.

The current analysis is part of a project to collect observational data from rheumatological patients followed at the ASST Gaetano Pini-CTO. The project was approved by the Ethics Committee of the Gaetano Pini Institute with approval number 141/2010. All included patients gave informed consent to participate in the data collection.

Address correspondence to Dr. E.G. Favalli, Division of Clinical Rheumatology, ASST Gaetano Pini-CTO Institute, Via Gaetano Pini 9, 20122 Milan, Italy. Email: enniofavalli@me.com.

\section{REFERENCES}

1. So H, Mak JW, Tam LS. No systemic lupus erythematosus with COVID-19 in Hong Kong: the effect of masking? J Rheumatol 2020;47:1591-2.

2. Favalli EG, Agape E, Caporali R. Incidence and clinical course of COVID-19 in patients with connective tissue diseases: a descriptive observational analysis. J Rheumatol 2020 May 15 (E-pub ahead of print).

3. Mathian A, Mahevas M, Rohmer J, Roumier M, Cohen-Aubart F, Amador-Borrero B, et al. Clinical course of coronavirus disease 2019 (COVID-19) in a series of 17 patients with systemic lupus erythematosus under long-term treatment with hydroxychloroquine. Ann Rheum Dis 2020;79:837-9.

4. Gao J, Tian Z, Yang X. Breakthrough: chloroquine phosphate has shown apparent efficacy in treatment of COVID-19 associated pneumonia in clinical studies. Biosci Trends 2020;14:72-3.

5. Mehra MR, Desai SS, Ruschitzka F, Patel AN. Hydroxychloroquine or chloroquine with or without a macrolide for treatment of COVID-19: a multinational registry analysis. Lancet 2020 May 22 (E-pub ahead of print).

6. Favalli EG, Gerosa M, Murgo A, Caporali R. Are patients with systemic lupus erythematosus at increased risk for COVID-19? Ann Rheum Dis 2020 May 25 (E-pub ahead of print).

7. Cassione EB, Zanframundo G, Biglia A, Codullo V, Montecucco C, Cavagna L. COVID-19 infection in a northern-Italian cohort of systemic lupus erythematosus assessed by telemedicine. Ann Rheum Dis 2020 May 12 (E-pub ahead of print).

8. Gianfrancesco MA, Hyrich KL, Gossec L, Strangfeld A, Carmona L, Mateus EF, et al. Rheumatic disease and COVID-19: initial data from the COVID-19 Global Rheumatology Alliance provider registries. Lancet Rheumatol 2020 Apr 16 (E-pub ahead of print). 